\title{
Association Analysis Between Common Variants of the TRPM1 Gene and Three Mental Disorders in the Han Chinese Population
}

\author{
Chuanchuan Ma, Xiuli Li, Jianhua Chen, ${ }^{1-4}$ Zhiqiang Li, ${ }^{1,3-5}$ \\ Jian Guan, ${ }^{3,4}$ Yigang Li, ${ }^{2,6,7}$ Shankai Yin, ${ }^{3,4}$ and Yongyong Shi ${ }^{1-7}$
}

Objective: Our study was designed to determine if the TRPM1 gene is associated with any of three mental disorders. The project included a cross disorder meta-analysis and association analysis including 141701 cases and 175248 controls.

Materials and Methods: We genotyped eight tag single nucleotide polymorphisms (SNPs) in 1248 unrelated schizophrenia (SCZ) patients, 1056 major depressive disorder patients, 1344 bipolar disorder patients, and 1248 normal controls. We then performed a meta-analysis of 10 GWASs to identify common genetic factors among these three mental disorders. Finally, we performed a meta-analysis of six GWASs to explore the role of rs10162727 in SCZ.

Result: Although two haplotypes of the TRPM1 gene, rs1035706-rs10162727 and rs10162727-rs3784599, were identified in the control group, as well as all three disease groups, none of the eight tag SNP associations remained significant after correction for multiple tests. In this cross-disorder meta-analysis of the three diseases, none of the tag SNPs were confirmed to be common among the diseases. In addition, in the meta-analysis conducted for the rs10162727 locus in SCZ, there was no significant association ( $p$-value $=0.84$, odds ratio $=1.02[95 \% \mathrm{CI}=0.87-1.19])$.

Conclusion: In the Han Chinese population, no significant evidence was found linking variants of the TRPMI gene with any of the mental disorders examined.

Keywords: TRPM1, schizophrenia, major depressive disorder, bipolar disorder, association study

\section{Introduction}

Q ChIZOPHRENIA (SCZ), MAJOR DEPRESSIVE DISORDER (MDD), and bipolar disorder (BPD) are known as complex polygenic psychiatric disorders. SCZ disables brain functions and could produce serious related negative effects on a patient's movement and cognitive functionthat is, in their daily life, as well as their social responsi- bilities. The traits of SCZ are divided into three categories: positive symptoms (hallucinations, delusions); negative symptoms (social withdrawal, reduced affective expression); and cognitive impairments (Karam et al., 2010). MDD is attributed to mental disease, which causes mental and long-term depression as an observational clinical symptom. BPD is characterized by alternating mania and depression.

\footnotetext{
${ }^{1}$ Department of Biology, School of Life Science, Anhui Medical University, Hefei, China.

${ }^{2}$ Shanghai Key Laboratory of Psychotic Disorders, Shanghai Mental Health Center, Shanghai Jiao Tong University School of Medicine, Shanghai Jiao Tong University, Shanghai, People's Republic of China.

${ }^{3}$ Department of Otolaryngology, Shanghai Jiao Tong University Affiliated Sixth People's Hospital, Shanghai, China.

${ }^{4}$ Department of Otolaryngology, Therapy Center for Obstructive Sleep Apnea, Otolaryngology Institute of Shanghai Jiao Tong University, Shanghai, China.

${ }^{5}$ The Affiliated Hospital of Qingdao University, The Biomedical Sciences Institute of Qingdao University (Qingdao Branch of SJTU Bio-X Institutes), Qingdao University, Qingdao, People's Republic of China.

${ }^{6}$ Department of Cardiology, Xinhua Hospital Affiliated to Shanghai Jiao Tong University School of Medicine, Shanghai, People's Republic of China.

${ }^{7}$ Bio-X Institutes, Key Laboratory for the Genetics of Developmental and Neuropsychiatric Disorders (Ministry of Education) and the Collaborative Innovation Center for Brain Science, Shanghai Jiao Tong University, Shanghai, People's Republic of China.

(C) Chuanchuan Ma et al., 2020; Published by Mary Ann Liebert, Inc. This Open Access article is distributed under the terms of the Creative Commons Attribution Noncommercial License (http://creativecommons.org/licenses/by-nc/4.0/) which permits any noncommercial use, distribution, and reproduction in any medium, provided the original author(s) and the source are cited.
} 
The lifetime prevalence of these three diseases in Han Chinese population was estimated at $0.66 \%$ (SCZ, 1994, mainland, China), 1.5\% (BPD, 2011, Shenzhen, China) (Merikangas et al., 2011), and 3.3\% (MDD, 2013, mainland China) (Gu et al., 2013). Although the pathogenic mechanisms of the three psychiatric disorders are still unclear, genetic factors definitely contribute to the pathophysiology of them. In essence, we established a cross-disease meta-analysis for finding the common genetic factors of the three diseases.

Neurotransmission makes an essential impact on neural circuit formation in the central nervous system (CNS). Neurotransmission has been recently clarified as a key modulator of CNS development; however, the roles of individual genes are not yet fully understood. There are types of protein that work as intermicellar transmission, including the transient receptor potential (TRP) superfamily. Currently, the TRP superfamily of ion channels contain more than 30 cationic channels, which are mostly permeable for $\mathrm{Ca}^{2+}$, but some are also for $\mathrm{Mg}^{2+}$ (Clapham, 2003). What is more, many TRP-related proteins were expressed in the nervous system (Montell et al., 2002).

The TRP box is located in the C-terminus domain of the TRPM family and no Ankyrin repeats in the N-terminus (Hantute-Ghesquier et al., 2018). According to the latest data obtained, the TRP domain was divided into two segments (Winkler et al., 2017). TRPM1 was found to be a member of the TRPM subfamily, and its sequence is similar to other members of the TRP family in the cation channels (Montell, 2005). The previous study indicates that TRPM1 works as a cation permeable protein, and as expressed in ON bipolar cells, it mediates neurotransmission between photoreceptors and ON bipolar cells (Koike et al., 2010).

Essentially, a visual signal is received by photoreceptors in the mammalian retina, and then the information is segregated into ON and OFF pathways (Kozuka et al., 2017). In current studies, Flash electroretinography (fERG) has been used to identify anomalies in the retinal cell function of patients with SCZ and major depression (Hebert et al., 2017; Demmin et al., 2018).

SCZ is a common mental illness with a large genetic component (Freedman et al., 2001; Owen et al., 2004). Significant or suggestive evidence linked to SCZ has implicated 18 chromosomal regions (Baron, 2001; Harrison and Owen, 2003). Twelve of these linked chromosomal regions have been replicated-including one or more polymorphisms such as SCZassociated polymorphisms - and contain biologically believable candidate genes showing altered expression in the disorder (Harrison and Weinberger, 2005). In addition, the 15q13-q14 locus is one of these regions, and its replication results are from the National Institute of Mental Health's (NIMH) SCZ Genetic Initiative (Freedman et al., 2001; Owen et al., 2004).

Notably, the results of a study by Stephens also indicated that $T R P M 1$ gene, along with $K L F 13$ and $R Y R 3$, was associated with $\mathrm{SCZ}$ in African Americans - that is, after correcting for multiple comparisons $\left(p\right.$-value $=4 \times 10^{-8}$, odds ratio $\left.=3.97\right)$ (Stephens et al., 2012). Moreover, TRPM1 was also identified as a candidate gene associated with symptoms of SCZ from genetic and mouse mutant model evidence (Forsingdal et al., 2016).

However, we found two pooling databases that show opposite consequences. In essence, pooling database (drawn from 2010 to 2011) concludes with data from four metaanalyses or genome-wide association studies (GWASs) per- taining to European ancestry, and then pooling database 2010-2013 concludes with data from five meta-analyses or GWASs in European ancestry as well (Cross-Disorder Group of the Psychiatric Genomics Consortium, 2013; Neale et al., 2010; Psychiatric GWAS Consortium Bipolar Disorder Working Group, 2011; Ripke et al., 2013; Schizophrenia Psychiatric Genome-Wide Association Study (GWAS) Consortium, 2011). Pooling database 2010-2011 indicates $p$-value $=0.402$ and Odds ratio $=0.884($ rs10162727 $)$ while database 2010-2013 indicates $p$-value $=0.334$ and Odds ratio $=1.295$ (rs10162727), which means that there is an insignificant association with SCZ (Neale et al., 2010; Ripke et al., 2013). However, we also noticed that, according to Ruderfer et al. (2014), GWASs pertaining to bipolar disorder and SCZ patients in the European population indicated that rs10162727 was not associated with SCZ $(p$-value $=0.5093$, Odds ratio $=1.072)$

These conflicting results motivate us to further verify in psychiatric disorders in Han Chinese population. In this study, we performed a cross-disorder association analysis of the TRPM1 gene in unrelated Han Chinese SCZ, MDD, and BPD patients-as well as normal controls - to verify previous findings and possibly discover new potential associations.

\section{Materials and Methods}

\section{Subjects}

Due to the inheritance factor of mental illness, age of onset in relation to psychiatrics, and also gender, we recruited 4896 unrelated Chinese subjects for our study. Among said subjects, 1248 were unrelated SCZ patients, 1056 were unrelated MDD cases, 1344 were unrelated bipolar disorder cases, and 1248 were normal controls (sample details are shown in Table 1).

All of the cases were interviewed by two independent psychiatrists who, subsequently, made a formal diagnosis that confirmed each subject's classification, which was in accordance with the Diagnostic and Statistical Manual of Mental Disorders Fourth Edition (DSM-IV). In the control groups, all subjects were not only randomly selected from the general population but also in a manner that ensured that all subjects were without family histories of psychiatric disorders. All participants knew the purpose and potential hazard of the study, and they signed written informed consent forms before their participation.

\section{Single nucleotide polymorphism selection}

According to results by Haploview 4.2 software, eight tag SNPs (rs12901022, rs1035706, rs10162727, rs3784599, rs10400821, rs4779503, rs12441329, rs12910440) that

Table 1. Detailed Information of the Sample Set

\begin{tabular}{lccccr}
\hline & \multicolumn{2}{c}{ Gender } & & \multicolumn{2}{c}{ Age (years) } \\
\cline { 2 - 3 } \cline { 5 - 6 } & Females & Males & & Average age & $S D$ \\
\hline Schizophrenia & 403 & 845 & & 36.44 & 9.00 \\
MDD & 319 & 737 & & 34.41 & 12.09 \\
BPD & 760 & 584 & & 34.84 & 11.44 \\
Normal controls & 576 & 672 & & 30.62 & 11.35 \\
\hline
\end{tabular}

MDD, major depressive disorder; BPD, bipolar disorder; SD, standard deviation. 
emerged were picked across the whole gene, which was accomplished using genotype data-of Han Chinese in Beijing, China (CHB) - from the International HapMap Project (Barrett et al., 2005) and rs10162727, which was defined as having single nucleotide polymorphism (SNP) susceptibility. Linkage disequilibrium $r^{2}$ threshold was set as 0.5 with a minor allele frequency that was greater than 0.05 (De Bakker et al., 2005, 2006). Next, a total of eight SNPs were selected for our study, and the locations are indicated in Figure 1.

\section{Genotyping}

The genomic DNA was extracted from peripheral blood by QuickGene DNA Whole Blood Kit (Fujifilm, Tokyo, Japan) and according to the manufacturer's agreement. Genotyping was performed using iPLEX chemistry on a matrix-assisted laser desorption/ionization time-of-flight mass spectrometer (MALDI-TOF-MS, named as the MassARRAY system, which is manufactured by Sequenom, San Diego, CA). Polymerase chain reaction (PCR) and extended primers were designed using the online primer design tool Sequenom. Mass spectrum was obtained using Compact Mass Spectrometer and analyzed with MassARRAY Typer 4.0 Software.

\section{Statistical analyses}

It was a basic component to ensure that Allele and genotype frequency calculation, pair-wise linkage disequilibrium analysis, Hardy-Weinberg $(\mathrm{H}-\mathrm{W})$ equilibrium analysis, and haplotype were all carried out on the SHEsisPlus online software platform in single site association study (Shen et al., 2016). H-W equilibrium was received through chi-square test for each group of fitness. Chi-square test independence was used to examine allele and genotype distribution between cases and controls. For haplotype analysis, in all cases and control groups, adjacent tag SNPs with pair-wise linkage disequilibrium $\mathrm{D}$ values larger than 0.90 were defined in the same haplotype block.

All statistical analyses were two-tailed tests, and if the $p$-values were below 0.05, Bonferroni correction ( $p$-value multiplied by the number of testing SNPs) should be set to test the statistical significance of results. Case-control genetic power was calculated on the Genetic Power Calculator (Purcell et al., 2003).

\section{Meta-analysis}

We used the keywords "TRPM1," "Schizophrenia," "Major Depressive Disorder," and "Bipolar Disorder"' to build a hunting zone in several databases-including PubMed, EMBASE, and Web of Science-to retrieve all pertinent studies or GWAS involving TRPM1 gene variants. In essence, we found a pooling database from Psychiatric Genomics Consortium (PGC) about SCZ. The collected data were combined with the statistical data from our study to perform a meta-analysis.

The meta-analysis procedures were conducted using Review Manager 5.3 software. Heterogeneity was assessed by the chi square and quantified by $I^{2}$ statistics, which ensured that the group of studies was suitable for meta-analysis. An odds ratio (OR) with a $95 \%$ confidence interval (CI) indicates the magnitude of the effect. The $\mathrm{Z}$ test was used as a means of estimating the $p$-value of OR. All $p$-values were reported as two-tailed tests, which would, ultimately, be regarded as significant statistic while determining whether or not the $p$-value was less than 0.05 .

\section{Results}

\section{Single site analysis}

According to export results from SHEsisPlus, all eight SNPs passed H-W equilibrium tests in control groups. The average call rate of all sites in three disease groups and controls was 0.981. Detailed information pertaining to these sites is supplemented in Table 2.

For SCZ, rs10400821 indicated genotypic or allelic association before Bonferroni correction (rs10400821: $\mathrm{P}_{\text {allele }}=0.564$, OR $[95 \% \mathrm{CI}]=1.178[0.934-1.485] ; \mathrm{P}_{\text {genotype }}=$ 0.022 , adjusted $P_{\text {genotype }}=0.182$ ). For BPD, rs4779503 was significant before multiple-test corrections (rs4779503: $\mathrm{P}_{\text {allele }}=0.256$, OR [95\% CI $]=1.167[0.893-1.526] ; \mathrm{P}_{\text {genotype }}=$ 0.023 , adjusted $\mathrm{P}_{\text {genotype }}=0.187$ ). For MDD, rs12901022 showed marginal significances in both allelic and genotypic analyses (rs12901022: $\mathrm{P}_{\text {allele }}=0.069$, OR $[95 \% \mathrm{CI}]=1.162$ [0.987-1.366]; $\left.\mathrm{P}_{\text {genotype }}=0.067\right)$. However, none of these associations remained after multiple tests and corrections. The results are listed in Table 3.

\section{Pairwise linkage disequilibrium analysis}

The pairwise linkage disequilibrium $\mathrm{D}$ values among the eight SNPs were calculated in control, as well as all three disease groups (Fig. 2). Relying upon the haplotype analysis criteria illustrated in the former section, the two blocks of TRPM1 and rs1035706-rs10162727 were identified in a control group, as well as all three disease groups. As such, rs10162727-rs3784599 was identified in a control group and, also, the BPD and SCZ groups.

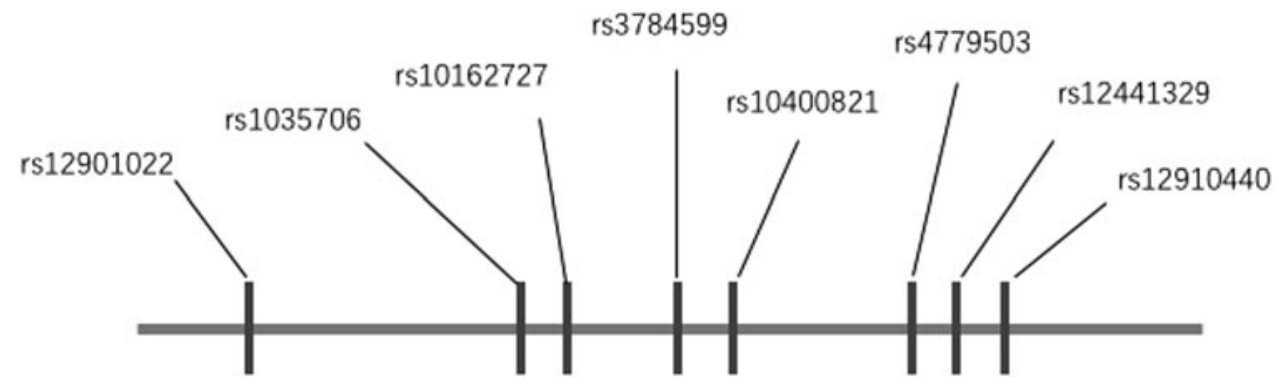

FIG. 1. Locations of the tag SNPs within the TRPM1 gene and all of that distributed across the whole gene. SNP, single nucleotide polymorphism. 
Table 2. Detailed Information of the Testing Single Nucleotide Polymorphisms in the TRPM1 Gene

\begin{tabular}{|c|c|c|c|c|c|c|}
\hline$S N P I D$ & Position & Function & Polymorphism & Group & Call rate & $H$-W $\mathrm{p}$-value \\
\hline \multirow[t]{4}{*}{ rs12901022 } & \multirow[t]{4}{*}{31020540} & \multirow[t]{4}{*}{ Intron } & \multirow[t]{4}{*}{$\mathrm{C} / \mathrm{T}$} & SCZ & 0.977 & 0.5 \\
\hline & & & & MDD & 0.99 & 0.321 \\
\hline & & & & BD & 0.989 & 0.633 \\
\hline & & & & Control & & 0.768 \\
\hline \multirow[t]{4}{*}{ rs1035706 } & \multirow[t]{4}{*}{31050882} & \multirow{4}{*}{ Intron } & \multirow{4}{*}{$\mathrm{A} / \mathrm{G}$} & SCZ & 0.977 & 0.111 \\
\hline & & & & MDD & 0.991 & 0.866 \\
\hline & & & & $\mathrm{BD}$ & 0.990 & 0.805 \\
\hline & & & & Control & & 0.84 \\
\hline \multirow{4}{*}{ rs 10162727} & \multirow{4}{*}{31051683} & \multirow{4}{*}{ Intron } & \multirow{4}{*}{$\mathrm{A} / \mathrm{G} / \mathrm{T}$} & SCZ & 0.975 & 0.999 \\
\hline & & & & MDD & 0.991 & 0.999 \\
\hline & & & & $\mathrm{BD}$ & 0.990 & 0.999 \\
\hline & & & & Control & & 0.999 \\
\hline \multirow{4}{*}{ rs3784599 } & \multirow{4}{*}{31067254} & \multirow{4}{*}{ Intron } & \multirow[t]{4}{*}{$\mathrm{G} / \mathrm{T}$} & SCZ & 0.994 & 0.863 \\
\hline & & & & MDD & 0.995 & 0.827 \\
\hline & & & & $\mathrm{BD}$ & 0.998 & 0.691 \\
\hline & & & & Control & & 0.993 \\
\hline \multirow[t]{4}{*}{ rs 10400821} & \multirow[t]{4}{*}{31069401} & \multirow[t]{4}{*}{ Intron \& UTR 3prime } & \multirow[t]{4}{*}{$\mathrm{A} / \mathrm{G}$} & SCZ & 0.973 & 0.013 \\
\hline & & & & MDD & 0.989 & 0.218 \\
\hline & & & & $\mathrm{BD}$ & 0.988 & 0.971 \\
\hline & & & & Control & & 0.519 \\
\hline \multirow{4}{*}{ rs4779503 } & \multirow[t]{4}{*}{31080131} & \multirow[t]{4}{*}{ Intron } & \multirow{4}{*}{$\mathrm{A} / \mathrm{G} / \mathrm{T}$} & SCZ & 0.975 & 0.762 \\
\hline & & & & MDD & 0.991 & 0.126 \\
\hline & & & & $\mathrm{BD}$ & 0.990 & 0.19 \\
\hline & & & & Control & & 0.31 \\
\hline \multirow[t]{4}{*}{ rs12441329 } & \multirow[t]{4}{*}{31085370} & \multirow[t]{4}{*}{ Intron } & \multirow[t]{4}{*}{$\mathrm{C} / \mathrm{T}$} & SCZ & 0.969 & 0.615 \\
\hline & & & & MDD & 0.973 & 0.79 \\
\hline & & & & $\mathrm{BD}$ & 0.950 & 0.645 \\
\hline & & & & Control & & 0.656 \\
\hline \multirow{4}{*}{ rs12910440 } & 31088989 & Intron & $\mathrm{A} / \mathrm{C} / \mathrm{G} / \mathrm{T}$ & $\mathrm{SCZ}$ & 0.962 & 0.315 \\
\hline & & & & MDD & 0.973 & 0.769 \\
\hline & & & & $\mathrm{BD}$ & 0.953 & 0.995 \\
\hline & & & & Control & & 0.693 \\
\hline
\end{tabular}

H-W P, H-W equilibrium $p$-value; SCZ, schizophrenia; SNP, single nucleotide polymorphism.

\section{Cross-disorder meta-analysis}

In this cross-disorder meta-analysis, we recruited pooling database 2010-2011, pooling database 2010-2013, and five GWAS as collecting databases to build a meta-analysis (Bipolar Disorder and Schizophrenia Working Group of the Psychiatric Genomics Consortium, 2018; CrossDisorder Group of the Psychiatric Genomics Consortium, 2013; Neale et al., 2010; Ripke et al., 2013; Ruderfer et al., 2014; Stahl et al., 2018; Schizophrenia Psychiatric Genome-Wide Association Study (GWAS) Consortium, 2011; Schizophrenia Working Group of the Psychiatric Genomics Consortium, 2014; Wray et al., 2018). We separately analyzed these SNPs for understanding common genetic mechanism. After analyzing, the result shows rs12910440 $(p$-value $=0.49$, Odds ratio $=0.97[95 \% \mathrm{CI}=0.89-1.06])$, rs10162727 ( $p$-value $=0.41, \quad$ Odds ratio $=0.89 \quad[95 \%$ $\mathrm{CI}=0.68-1.17]), \quad$ rs10400821 $(p$-value $=0.26$, Odds ratio $=0.81[95 \% \mathrm{CI}=0.56-1.16])$, rs12901022 $(p$-value $=0.49$, Odds ratio $=0.76[95 \% \mathrm{CI}=0.35-1.65]), \operatorname{rs} 4779503 \quad(p$ value $=0.44$, Odds ratio $=0.80 \quad[95 \% \quad \mathrm{CI}=0.45-1.42])$, rs3784599 $(p$-value $=0.34$, Odds ratio $=0.77[95 \% \mathrm{CI}=0.44$ 1.32] $), \quad$ rs12441329 $\quad(p$-value $=0.51, \quad$ Odds $\quad$ ratio $=0.98$ $[95 \% \mathrm{CI}=0.91-1.05])$, and rs1035706 $(p$-value $=0.68$, Odds ratio $=0.99 \quad[95 \% \quad \mathrm{CI}=0.95-1.03]) \quad$ (Supplementary Figs. S1-S8).

\section{Meta-analysis for rs10162727 in SCZ}

We recruited pooling database 2010-2011 and pooling database 2010-2013 from PGC and, also, a GWAS about SCZ in which experimented SNPs overlapped with ours in the meta-analysis (Cross-Disorder Group of the Psychiatric Genomics Consortium, 2013; Neale et al., 2010; Ripke et al., 2013; Ruderfer et al., 2014; Schizophrenia Psychiatric Genome-Wide Association Study (GWAS) Consortium, 2011). After further consideration of studies performed in Han Chinese population, it was determined that rs10162727 was suitable for the meta-analysis. Merging all data, we suggest that there is no significant association between rs 10162727 and SCZ $(p$-value $=0.84$, Odds ratio $=1.02[95 \% \mathrm{CI}=0.87-1.19])$. The result of metaanalysis supports our results in this study of the Han Chinese population (Fig. 3).

\section{Discussion}

Currently, the pathogenesis of these three psychiatrics is still unclear. Family-based association studies also show comorbidity among BPD and MDD (Smoller and Finn, 2003), as well as SCZ and BPD (Valles et al., 2000). These three diseases have general characteristic symptoms, shared genetic etiology, and increased numbers of variants and gene 


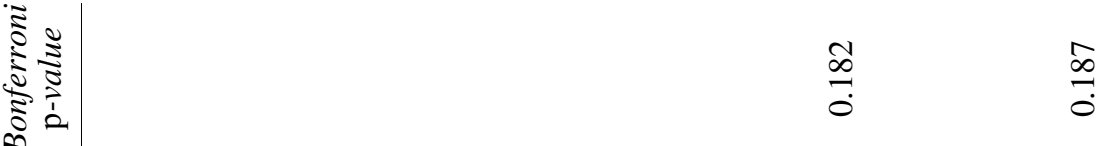

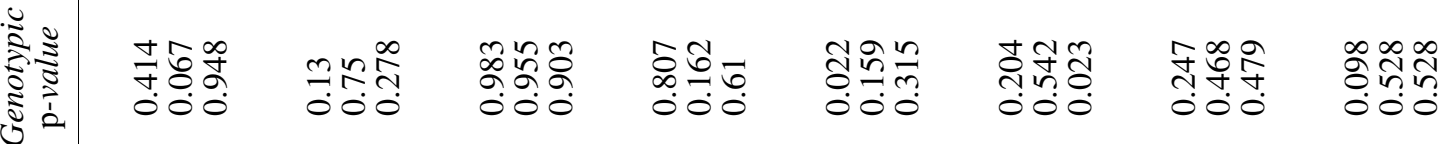

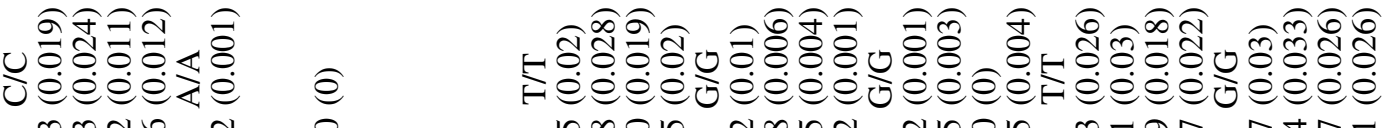

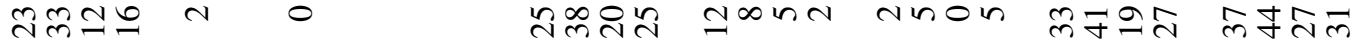

ड़े

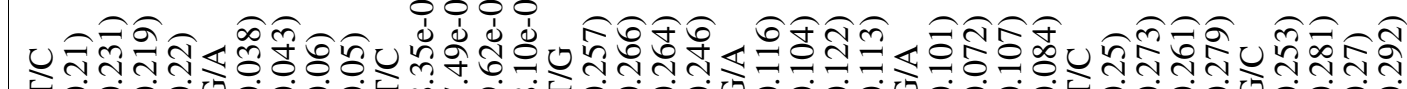

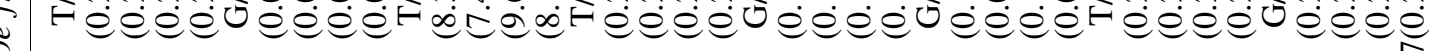

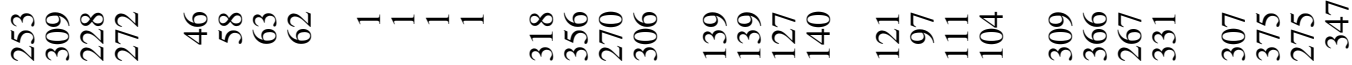

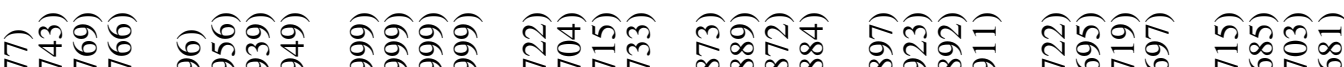

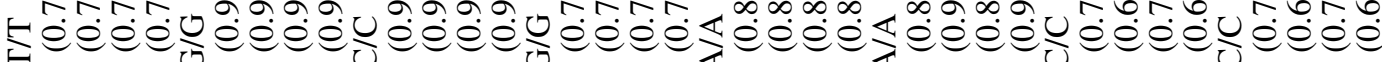

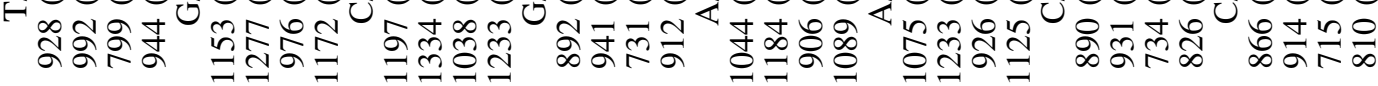

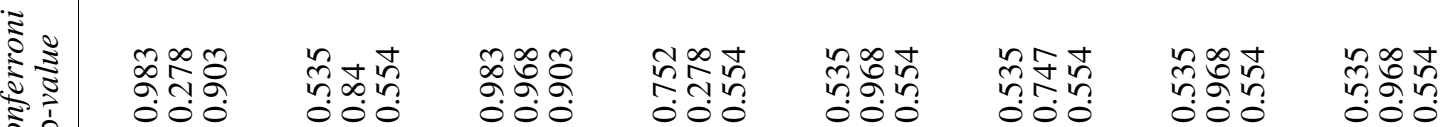

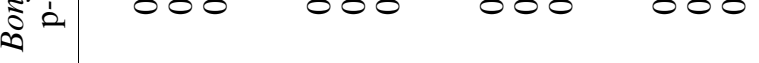

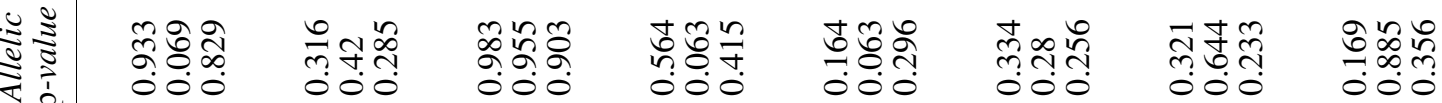

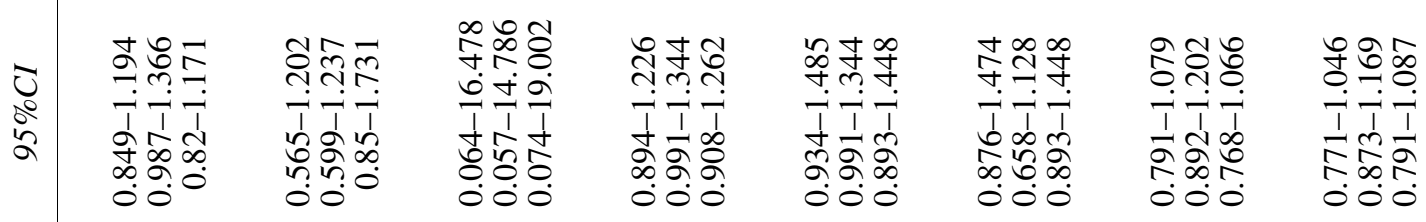

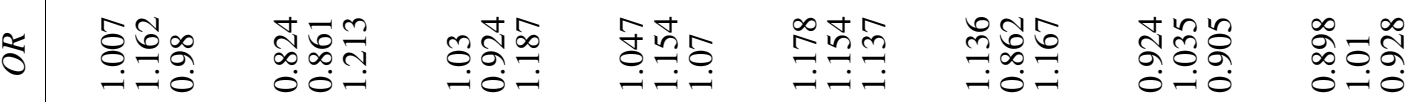

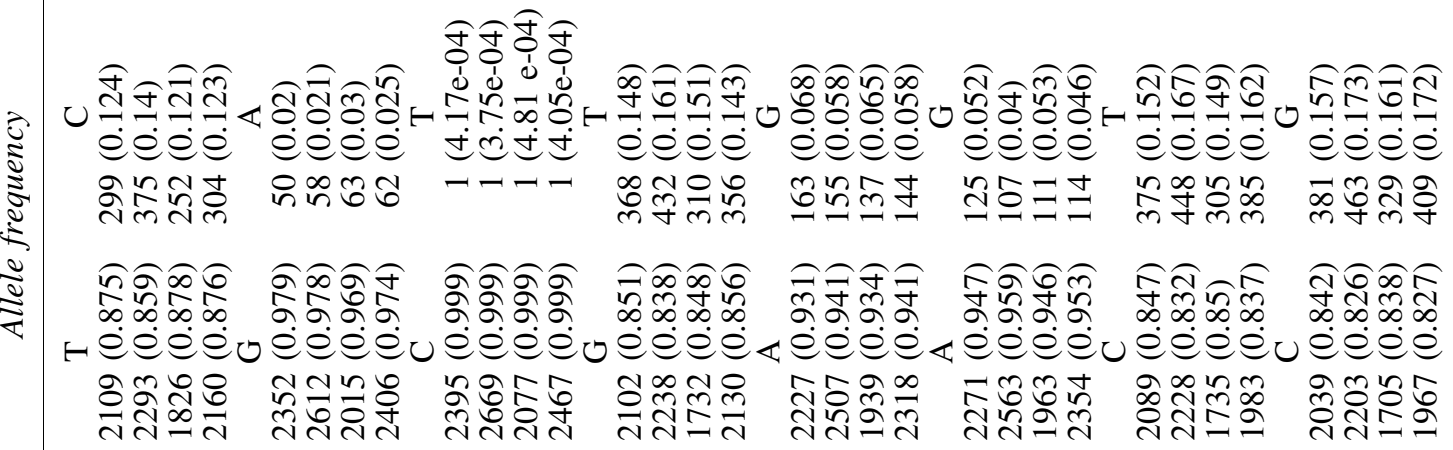

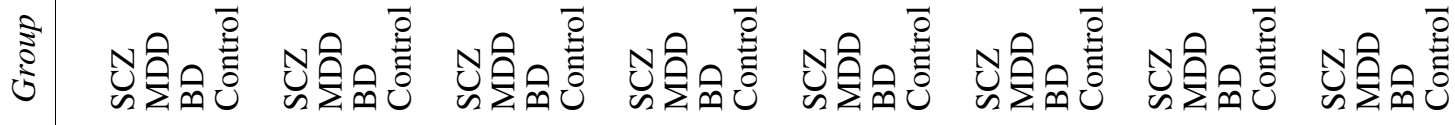

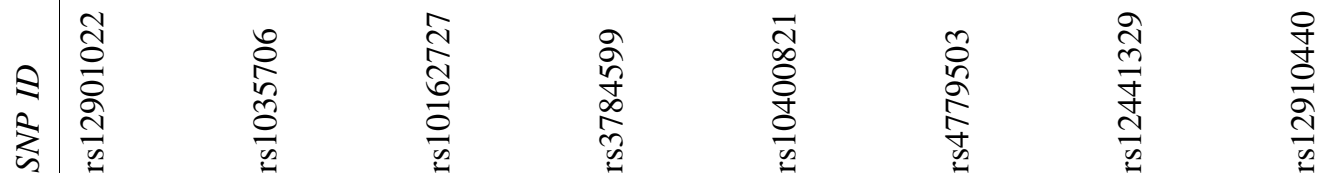




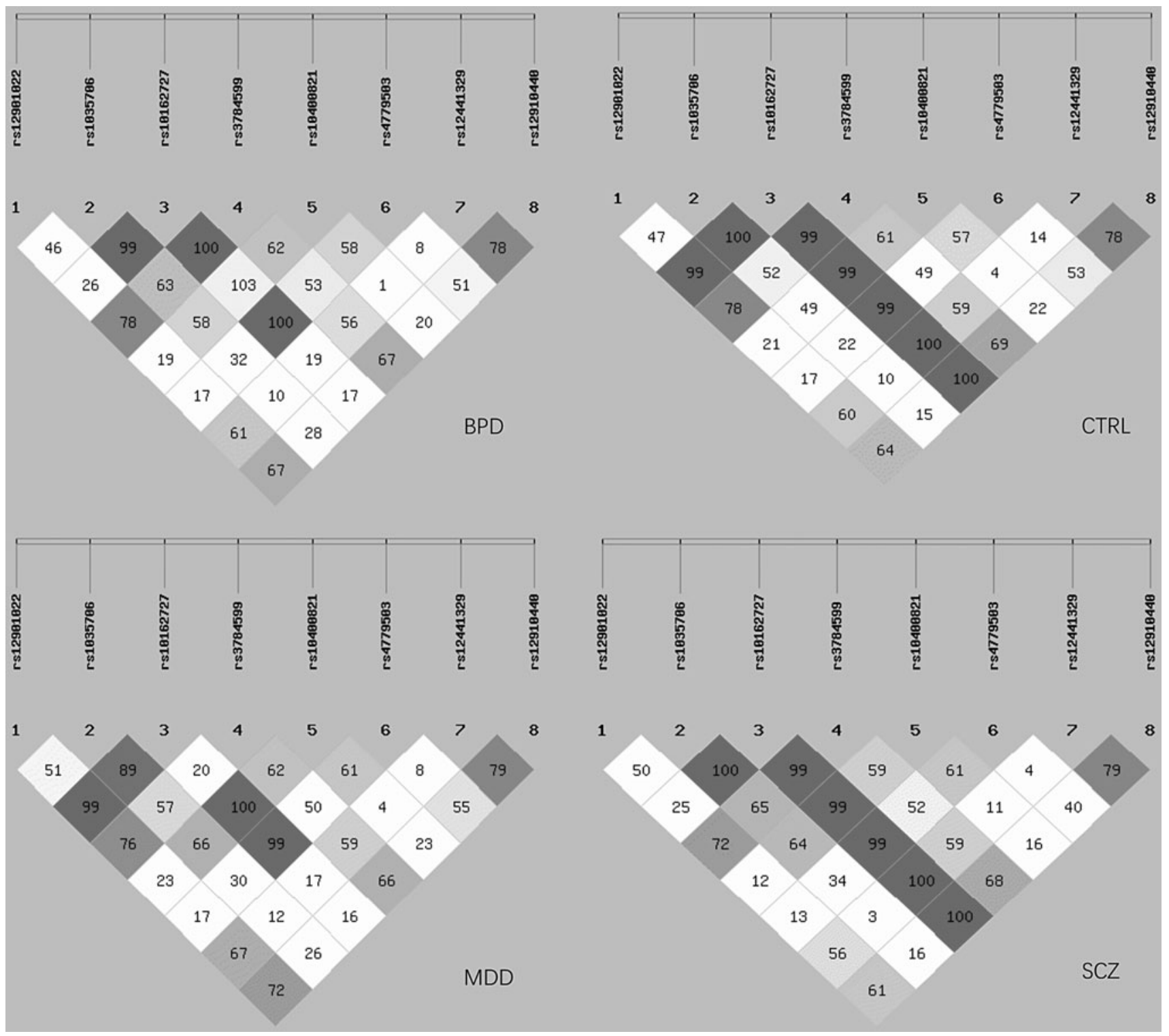

FIG. 2. Linkage disequilibrium among the eight SNPs in CTRL, SCZ, MDD, and BPD groups. The pairwise D'values are presented in the matrices. Dark gray implicates relatively strong linkage disequilibrium, and vice versa. CTRL, control; MDD, major depressive disorder; BPD, bipolar disorder; SCZ, schizophrenia.

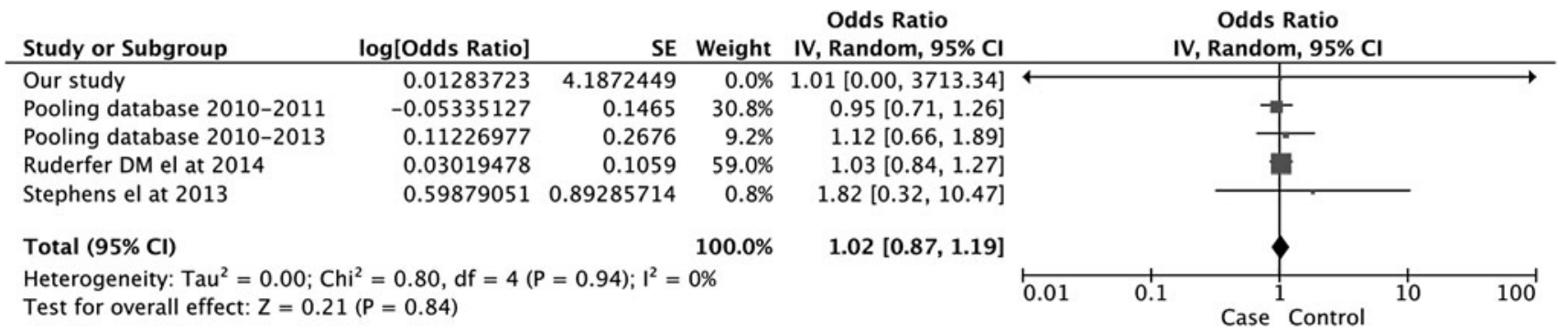

FIG. 3. Forest plot of the association between rs10162727 and schizophrenia in a random effects model. The point represents the OR, and horizontal lines indicate 95\% CI for each study. The diamond illustrates that summary OR and 95\% CI by random effect calculations. 
regions, such as: rs1344706 in the ZNF804A gene (Williams et al., 2011); rs1006737 in the CACNA1C gene also confers risk of recurrent MDD and SCZ (Green et al., 2010); and intron 42 of the $M Y O 18 B$ gene and intron 1 of the NPAS3 gene (Huang et al., 2010). Meanwhile, a previous cross-disorder GWAS revealed that common genetic factors can be shared among SCZ, MDD, and BPD (Ruderfer et al., 2014).

However, it is possible that similar genetic overlap relating to TRPM1 happens in major psychiatric illnesses of the Han Chinese population, especially in SCZ, MDD, and BPD. It is notable that SCZ, MDD, and BPD share many clinical symptoms and sign change. Due to the complicated pathogenesis of mental diseases, one of the obstacles in psychiatric exploration that is too difficult to dynamically analyze is the state of human brain.

Several retina dysfunctions have been observed in psychiatric patients through the fERG, and it is suggested that retinal activity may reflect uncommon neurochemistry in brain disorders (Lavoie et al., 2014). Forsingdal built a 15q13.3 homozygous knockout mouse model effect using seven genes-FAN1, MTMR10, TRPM1, miR-211, KLF13, OTUD7A, and CHRNA7-and the study suggests that the cognitive disabilities of Df (h15q13) ${ }^{-1-}$ mice were related to SCZ (Forsingdal et al., 2016). Thus, this could be the reason for why cognitive disabilities may cause visual impairments-that is, because the TRPM1 gene has been linked to deficits in visual light response (Morgans et al., 2009; Koike et al., 2010; Hughes et al., 2012; Kozuka et al., 2017).

Previously, it was reported that TRPM1 showed a significant association with SCZ in African Americans (105 cases and 45 controls) (Stephens et al., 2012), but we also found opposite results in some GWASs that considered multiple races (Ruderfer et al., 2014) (Bipolar Disorder and Schizophrenia Working Group of the Psychiatric Genomics Consortium, 2018; Schizophrenia Psychiatric Genome-Wide Association Study (GWAS) Consortium, 2011; Schizophrenia Working Group of the Psychiatric Genomics Consortium, 2014; Stahl et al., 2018; Wray et al., 2018). Because of the conflicting conclusions between these different studies, we searched an imputed dataset of a Zhiqiang et al. (2017) study that was published in 2017 to find out whether or not TRPMI was associated with these three diseases. However, the results suggest insignificant association between SCZ and TRPM1, and these tag SNPs were not found in the imputed database.

It is well known that the three diseases have similar clinical signs. What is more, recent genetic and epidemiological studies have demonstrated substantial overlap between these three disorders (Cross-Disorder Group of the Psychiatric Genomics C, 2013). Meta-analysis can improve the efficiency of research and further discover the relationship between the three diseases. Only then can we combine patients with SCZ or BPD or MDD for crossdisorder genetic research analysis. In this cross-disorder meta-analysis of the diseases, all tag SNPs fail to confirm that there are cross-disorder common genetic factors. In addition, in meta-analysis for rs 10162727 in SCZ, we still suggested that there was no significant association between rs10162727 and SCZ ( $p$-value $=0.84$, Odds ratio $=1.02$ $[95 \% \mathrm{CI}=0.87-1.19])$.
All of the SNPs failed to prove statistically significant associations with SCZ, MDDs, and bipolar disorders by the negative result of the meta-analysis. This means TRPM1 did not play a role in the three psychiatric diseases within Han Chinese population. Alas, research on disease is infinite, so more research is expected to contribute to the exploration of disease occurrence and development.

\section{Author Disclosure Statement}

No competing financial interests exist.

\section{Funding Information}

This work was supported by the National Key R\&D Program of China (2017YFC0908105), National Key R\&D Program of China (2019YFA0905400), Shanghai Municipal Science and Technology Major Project (2017SHZDZX01), the Natural Science Foundation of China (U1804284, 81421061, 81701321, 31571012, 81501154), the Program of Shanghai Subject Chief Scientist (15XD1502200), the National Program for Support of Top-Notch Young Professionals, Shanghai Key Laboratory of Psychotic Disorders (13dz2260500), the National Program for Support of TopNotch Young Professionals to Y.S., Shanghai Hospital Development Center (SHDC12016115), Shanghai Municipal Commission of Science and Technology (17JC1402900, 17490712200, 18DZ2260200) and Shanghai Municipal Health Commission (ZK2015B01, 201540114), and Scientific Research and Development Fund of Shanghai Jiao Tong University (19X150010012).

\section{Supplementary Material}

Supplementary Figure S1 Supplementary Figure S2 Supplementary Figure S3 Supplementary Figure S4 Supplementary Figure S5 Supplementary Figure S6 Supplementary Figure S7 Supplementary Figure S8

\section{References}

Baron M. (2001) Genetics of schizophrenia and the new millennium: progress and pitfalls. Am J Hum Genet 68: 299-312.

Barrett JC, Fry B, Maller J, et al. (2005) Haploview: analysis and visualization of LD and haplotype maps. Bioinformatics 21:263-265.

Bipolar Disorder and Schizophrenia Working Group of the Psychiatric Genomics Consortium. (2018) Genomic Dissection of Bipolar Disorder and Schizophrenia, Including 28 Subphenotypes. Cell 173:1705-1715.e1716.

Clapham DE (2003) TRP channels as cellular sensors. Nature 426:517-524.

Cross-Disorder Group of the Psychiatric Genomics Consortium (2013) Identification of risk loci with shared effects on five major psychiatric disorders: a genome-wide analysis. Lancet 381:1371-1379. 
Cross-Disorder Group of the Psychiatric Genomics Consortium, Hong LS, Stephan R, et al. (2013) Genetic relationship between five psychiatric disorders estimated from genome-wide SNPs. Nat Genet 45:984-994.

De Bakker PI, Yelensky R, Pe'er I, et al. (2005) Efficiency and power in genetic association studies. Nat Genet 37:12171223.

De Bakker PI., Burtt NP, Graham RR, et al. (2006) Transferability of tag SNPs in genetic association studies in multiple populations. Nat Genet 38:1298-1303.

Demmin DL, Davis Q, Roche M, et al. (2018) Electroretinographic anomalies in schizophrenia. J Abnorm Psychol 127: 417-428.

Forsingdal A, Fejgin K, Nielsen V, et al. (2016) 15q13.3 homozygous knockout mouse model display epilepsy-, autism- and schizophrenia-related phenotypes. Transl Psychiatry 6:e860.

Freedman R, Leonard S, Olincy A, et al. (2001) Evidence for the multigenic inheritance of schizophrenia. Am J Med Genet 105:794-800.

Green EK, Grozeva D, Jones I, et al. (2010) The bipolar disorder risk allele at CACNA1C also confers risk of recurrent major depression and of schizophrenia. Mol Psychiatry 15: 1016-1022.

Gu L, Xie J, Long J, et al. (2013) Epidemiology of major depressive disorder in mainland china: a systematic review. PLoS One 8:e65356.

Hantute-Ghesquier A, Haustrate A, Prevarskaya N, et al. (2018) TRPM family channels in cancer. Pharmaceuticals (Basel) 11:58.

Harrison PJ, Owen MJ (2003) Genes for schizophrenia? Recent findings and their pathophysiological implications. Lancet 361:417-419.

Harrison PJ, Weinberger DR (2005) Schizophrenia genes, gene expression, and neuropathology: on the matter of their convergence. Mol Psychiatry 10:40-68; image 45.

Hebert M, Merette C, Paccalet T, et al. (2017) Electroretinographic anomalies in medicated and drug free patients with major depression: tagging the developmental roots of major psychiatric disorders. Prog Neuropsychopharmacol Biol Psychiatry 75:10-15.

Huang J, Perlis RH, Lee PH, et al. (2010) Cross-disorder genomewide analysis of schizophrenia, bipolar disorder, and depression. Am J Psychiatry 167:1254-1263.

Hughes S, Pothecary CA, Jagannath A, et al. (2012) Profound defects in pupillary responses to light in TRPM-channel null mice: a role for TRPM channels in non-image-forming photoreception. Eur J Neurosci 35:34-43.

Karam CS, Ballon JS, Bivens NM, et al. (2010) Signaling pathways in schizophrenia: emerging targets and therapeutic strategies. Trends Pharmacol Sci 31:381-390.

Koike C, Obara T, Uriu Y, et al. (2010) TRPM1 is a component of the retinal ON bipolar cell transduction channel in the mGluR6 cascade. Proc Natl Acad Sci U S A 107:332337.

Kozuka T, Chaya T, Tamalu F, et al. (2017) The TRPM1 Channel Is Required for Development of the Rod ON Bipolar Cell-AII amacrine cell pathway in the retinal circuit. J Neurosci 37:9889-9900.

Lavoie J, Maziade M, Hebert M (2014) The brain through the retina: the flash electroretinogram as a tool to investigate psychiatric disorders. Prog Neuropsychopharmacol Biol Psychiatry 48:129-134.
Merikangas KR, Jin R, He JP, et al. (2011) Prevalence and correlates of bipolar spectrum disorder in the world mental health survey initiative. Arch Gen Psychiatry 68: 241-251.

Montell C (2005) The TRP superfamily of cation channels. Sci STKE 2005:re3.

Montell C, Birnbaumer L, Flockerzi V (2002) The TRP channels, a remarkably functional family. Cell 108:595598.

Morgans CW, Zhang J, Jeffrey BG, et al. (2009) TRPM1 is required for the depolarizing light response in retinal ON-bipolar cells. Proc Natl Acad Sci U S A 106:1917419178.

Neale BM, Medland SE, Ripke S, et al. (2010) Meta-analysis of genome-wide association studies of attention-deficit/ hyperactivity disorder. J Am Acad Child Adolesc Psychiatry 49:884-897.

Owen MJ, Williams NM, O'donovan MC (2004) The molecular genetics of schizophrenia: new findings promise new insights. Mol Psychiatry 9:14-27.

Psychiatric GWAS Consortium Bipolar Disorder Working Group (2011) Large-scale genome-wide association analysis of bipolar disorder identifies a new susceptibility locus near ODZ4. Nat Genet 43:977-983.

Purcell S, Cherny SS, Sham PC (2003) Genetic Power Calculator: design of linkage and association genetic mapping studies of complex traits. Bioinformatics 19:149-150.

Ripke S, Wray NR, Lewis CM, et al. (2013) A mega-analysis of genome-wide association studies for major depressive disorder. Mol Psychiatry 18:497-511.

Ruderfer DM, Fanous AH, Ripke S, et al. (2014) Polygenic dissection of diagnosis and clinical dimensions of bipolar disorder and schizophrenia. Mol Psychiatry 19: 1017-1024.

Schizophrenia Psychiatric Genome-Wide Association Study (GWAS) Consortium (2011) Genome-wide association study identifies five new schizophrenia loci. Nat Genet 43: 969-976.

Schizophrenia Working Group of the Psychiatric Genomics Consortium (2014) Biological insights from 108 schizophreniaassociated genetic loci. Nature 511:421-427.

Shen J, Li Z, Chen J, et al. (2016) SHEsisPlus, a toolset for genetic studies on polyploid species. Sci Rep 6:24095.

Smoller JW, Finn CT (2003) Family, twin, and adoption studies of bipolar disorder. Am J Med Genet C Semin Med Genet $123 \mathrm{c}: 48-58$.

Stahl EA, Gerome B, Andreas F, et al. (2018) Genomewide association study identifies 30 loci associated with bipolar disorder. Nat Genet 51:793-803.

Stephens SH, Franks A, Berger R, et al. (2012) Multiple genes in the 15q13-q14 chromosomal region are associated with schizophrenia. Psychiatr Genet 22:1-14.

Valles V, Van Os J, Guillamat R, et al. (2000) Increased morbid risk for schizophrenia in families of in-patients with bipolar illness. Schizophr Res 42:83-90.

Williams HJ, Norton N, Dwyer S, et al. (2011) Fine mapping of ZNF804A and genome-wide significant evidence for its involvement in schizophrenia and bipolar disorder. Mol Psychiatry $16: 429-441$.

Winkler PA, Huang Y, Sun W, et al. (2017) Electron cryomicroscopy structure of a human TRPM4 channel. Nature 552:200-204. 
Wray NR, Ripke S, Mattheisen M, et al. (2018) Genome-wide association analyses identify 44 risk variants and refine the genetic architecture of major depression. Nat Genet 50:668-681.

Zhiqiang L, Jianhua C, Hao Y, et al. (2017) Genome-wide association analysis identifies 30 new susceptibility loci for schizophrenia. Nat Genet 49:1576-1583.

Address correspondence to: Yongyong Shi, PhD Bio-X Institutes

Key Laboratory for the Genetics of Developmental and Neuropsychiatric Disorders (Ministry of Education) and the Collaborative Innovation Center for Brain Science

Shanghai Jiao Tong University Shanghai 200000 People's Republic of China

E-mail: shiyongyong@gmail.com
Shankai Yin, PhD

Department of Otolaryngology Shanghai Jiao Tong University Affiliated Sixth

People's Hospital

Shanghai 200000

China

E-mail: yinshankai@china.com

Yigang Li, PhD

Shanghai Key Laboratory of Psychotic Disorders Shanghai Mental Health Center Shanghai Jiao Tong University School of Medicine Shanghai Jiao Tong University

Shanghai 200000 People's Republic of China

E-mail: liyigang@xinhuamed.com.cn 